\title{
Obesity, inflammatory markers, and endometrial cancer risk: a prospective case-control study
}

\author{
Laure Dossus ${ }^{1}$, Sabina Rinaldi ${ }^{2}$, Susen Becker ${ }^{1}$, Annekatrin Lukanova ${ }^{1,3}$, \\ Anne Tjonneland ${ }^{4}$, Anja Olsen ${ }^{4}$, Jakob Stegger '5, Kim Overvad ${ }^{5,6}$, \\ Nathalie Chabbert-Buffet ${ }^{7}$, Aida Jimenez-Corona ${ }^{8,9}$, Francoise Clavel-Chapelon ${ }^{8}$, \\ Sabine Rohrmann ${ }^{1}$, Birgit Teucher ${ }^{1}$, Heiner Boeing ${ }^{10}$, Madlen Schütze ${ }^{10}$, \\ Antonia Trichopoulou ${ }^{11,12}$, Vassiliki Benetou ${ }^{11}$, Pagona Lagiou ${ }^{11}$, \\ Domenico Palli ${ }^{13}$, Franco Berrino ${ }^{14}$, Salvatore Panico ${ }^{15}$, Rosario Tumino ${ }^{16}$, \\ Carlotta Sacerdote ${ }^{17,18}$, Maria-Luisa Redondo ${ }^{19}$, Noémie Travier ${ }^{20}$, \\ Maria-Jose Sanchez 21,22, Jone M Altzibar 22,23 'Maria-Dolores Chirlaque 22,24 \\ Eva Ardanaz 22,25, H Bas Bueno-de-Mesquita 26, Fränzel J B van Duijnhoven '26, \\ N Charlotte Onland-Moret ${ }^{27}$, Petra HM Peeters' ${ }^{27}$, Goran Hallmans ${ }^{28}$, \\ Eva Lundin ${ }^{29}$, Kay-Tee Khaw ${ }^{30}$, Nicholas Wareham ${ }^{31}$, Naomi Allen ${ }^{32}$, \\ Tim J Key ${ }^{32}$, Nadia Slimani ${ }^{2}$, Pierre Hainaut ${ }^{33}$, Dora Romaguera ${ }^{34}$, \\ Teresa Norat $^{34}$, Elio Riboli ${ }^{34}$ and Rudolf Kaaks ${ }^{1}$
}

\footnotetext{
${ }^{1}$ Division of Cancer Epidemiology, German Cancer Research Center (DKFZ), Deutsches Krebsforschungszentrum, Im Neuenheimer Feld 280, 69120 Heidelberg, Germany

${ }^{2}$ Section of Nutrition and Metabolism, International Agency for Research on Cancer, 69372 Lyon, France

${ }^{3}$ Department of Obstetrics and Gynecology, New York University School of Medicine, 10016 New York, New York, USA

${ }^{4}$ Institute of Cancer Epidemiology, Danish Cancer Society, 2100 Copenhagen, Denmark

${ }^{5}$ Department of Cardiology, Aalborg Hospital, Aarhus University Hospital, 9100 Aalborg, Denmark

${ }^{6}$ Department of Epidemiology, School of Public Health, Aarhus University, 8000 Aarhus, Denmark

${ }^{7}$ Obstetrics and Gynecology Department, APHP Hospital Tenon and UMPC, 75970 Paris, France

${ }^{8}$ Inserm, Center for Research in Epidemiology and Population Health, Paris South University, Gustave Roussy Institut, 94805 Villejuif, France

${ }^{9}$ National Institute of Public Health, 62100 Cuernavaca, Morelos, Mexico

${ }^{10}$ Department of Epidemiology, German Institute of Human Nutrition Potsdam-Rehbruecke, 14558 Nuthetal, Germany

${ }^{11}$ WHO Collaborating Center for Food and Nutrition Policies, Department of Hygiene, Epidemiology and Medical Statistics, University of Athens Medical School, 11527 Athens, Greece

${ }^{12}$ Hellenic Health Foundation, 11527 Athens, Greece

${ }^{13}$ Molecular and Nutritional Epidemiology Unit, Cancer Research and Prevention Institute (ISPO), 50139 Florence, Italy

${ }^{14}$ Epidemiology Unit, Department of Preventive and Predicitive Medicine, Fondazione IRCCS Istituto Nazionale Tumori, 20139 Milan, Italy

${ }^{15}$ Department of Clinical and Experimental Medicine, Federico II University, 80138 Naples, Italy

${ }^{16}$ Cancer Registry and Histopathology Unit, 'Civile - M.P. Arezzo' Hospital, 97100 Ragusa, Italy

${ }^{17}$ Center for Cancer Prevention (CPO Piedmont), 10123 Turin, Italy

${ }^{18}$ Human Genetic Foundation (Hugef), 10126 Turin, Italy

${ }^{19}$ Public Health and Participation Directorate, Health and Health Care Services Council, 33001 Oviedo, Asturias, Spain

${ }^{20}$ Unit of Nutrition, Environment and Cancer, Cancer Epidemiology Research Programme, Catalan Institute of Oncology (ICO),

08907 Barcelona, Spain

${ }^{21}$ Andalusian School of Public Health, 18011 Granada, Spain

${ }^{22}$ CIBER Epidemiologia y Salud Publica (CIBERESP), 08003 Barcelona, Spain

${ }^{23}$ Department of Public Health of Guipuzkoa, 20013 San Sebastian, Spain

${ }^{24}$ Department of Epidemiology, Murcia Regional Health Authority, 30008 Murcia, Spain

${ }^{25}$ Public Health Institute of Navarra, 31003 Pamplona, Spain

${ }^{26}$ National Institute for Public Health and the Environment (RIVM), 3720 BA Bilthoven, The Netherlands

${ }^{27}$ Julius Center for Health Sciences and Primary Care, University Medical Center, 3508 GA Utrecht, The Netherlands

${ }^{28}$ Department of Public Health and Clinical Medicine, Nutritional Research, Umea University, 90187 Umea, Sweden

${ }^{29}$ Department of Medical Biosciences, Pathology, Umea University, 90187 Umea, Sweden

${ }^{30}$ Department of Public Health and Primary Care, University of Cambridge, Cambridge CB1 8RN, UK

${ }^{31}$ MRC Epidemiology Unit, Cambridge CB2 OQQ, UK
} 


\footnotetext{
${ }^{32}$ Cancer Epidemiology Unit, Nuffield Department of Clinical Medicine, University of Oxford, Oxford OX3 7XP, UK

${ }^{33}$ Section of Mechanisms of Carcinogenesis, International Agency for Research on Cancer, 69372 Lyon, France

${ }^{34}$ Department of Epidemiology and Biostatistics, School of Public Health, Imperial College, London SW7, UK

(Correspondence should be addressed to R Kaaks; Email: r.kaaks@dkfz.de)
}

\begin{abstract}
Obesity, a major risk factor for endometrial cancer, is a low-grade inflammatory state characterized by elevated concentrations of cytokines and acute phase reactants. The current study had two aims: first to investigate the associations of C-reactive protein (CRP), interleukin 6 (IL6), and IL1 receptor antagonist (IL1Ra) with endometrial cancer risk and second to examine to which extent these markers can influence the association between obesity and endometrial cancer. We conducted a case-control study, nested within the European Prospective Investigation into Cancer and Nutrition, which comprised 305 incident cases of endometrial cancer and 574 matched controls. CRP, IL6, and IL1Ra were measured in prospectively collected blood specimens by immunoassays. Data were analyzed using conditional logistic regression. All statistical tests were two-sided, and $P$ values $<0.05$ were considered statistically significant. We observed a significant increase in risk of endometrial cancer with elevated levels of CRP (odds ratio (OR) for top versus bottom quartile: $1.58,95 \%$ confidence interval $\left.(\mathrm{Cl}): 1.03-2.41, P_{\text {trend }}=0.02\right)$, IL6 (OR for top versus bottom quartile: $1.66,95 \% \mathrm{Cl}: 1.08-2.54, P_{\text {trend }}=0.008$ ), and IL1Ra (OR for top versus bottom quartile: $\left.1.82,95 \% \mathrm{Cl}: 1.22-2.73, P_{\text {trend }}=0.004\right)$. After adjustment for body mass index (BMI), the estimates were strongly reduced and became non-significant. The association between BMI and endometrial cancer was also substantially attenuated $(\sim 10-20 \%)$ after adjustment for inflammatory markers, even when the effects of C-peptide or estrone had already been taken into account. We provided epidemiological evidence that chronic inflammation might mediate the association between obesity and endometrial cancer and that endometrial carcinogenesis could be promoted by an inflammatory milieu.
\end{abstract}

Endocrine-Related Cancer (2010) 17 1007-1019

\section{Introduction}

Excess body weight is one of the strongest risk factors for endometrial cancer and accounts for about 50\% of the cases in Europe and in the USA (International Agency for Research on Cancer (IARC) 2002, Calle \& Kaaks 2004, World Cancer Research Fund/American Institute for Cancer Research 2007). Obese women (body mass index $(\mathrm{BMI})>30 \mathrm{~kg} / \mathrm{m}^{2}$ ) have a threefold increased risk of developing endometrial cancer compared to normal weight women (Calle \& Kaaks 2004, Reeves et al. 2007).

Several biological mechanisms could mediate the association between obesity and endometrial cancer risk. In postmenopausal women, excess weight leads, through peripheral conversion of androgens, to increased circulating bioavailable estrogens which in turn, when not counterbalanced by progesterone, increases the mitogenic activity of endometrial cells (Key \& Pike 1988). In premenopausal women, obesity has been associated with the development of polycystic ovary syndrome, a disease that affects $4-8 \%$ of women and is characterized by ovarian hyperandrogenism, anovulation, and progesterone deficiency (Ehrmann et al. 1995, Franks 1995). Obesity-associated hyperinsulinemia might also play an important role in endometrial carcinogenesis, in both pre and postmenopausal women, either directly, by stimulating endometrial cell proliferation, or indirectly, through the sex steroid and the insulin-like growth factor 1 pathways (Kaaks et al. 2002).

More recently, it has been proposed that other adiposity-related factors such as cytokines and adipokines might contribute to endometrial cancer initiation and progression and to the obesity-related increase in risk (Modugno et al. 2005). Excess body weight is associated with a systemic low-grade inflammatory condition (Das 2001), characterized by elevations in circulating pro-inflammatory cytokines and acute phase proteins (Mohamed-Ali et al. 1997, Visser et al. 1999, Yudkin et al. 1999, Meier et al. 2002). Chronic inflammation promotes angiogenesis, sustains cell proliferation, and increases production of free radicals that cause DNA damage, leading to tumor initiation and development (Coussens \& Werb 2002). Inflammatory processes also play a central role in the regulation of endometrial mucosa growth and shedding during the menstrual cycle (Kelly et al. 2001) as well as in endometrial repair following menstruation (Salamonsen 2003). 
C-reactive protein (CRP) is an acute phase protein produced by the liver in response to tissue damage and inflammation. CRP binds to damaged cellular tissues and components to facilitate phagocytosis and clearance by macrophages and leukocytes (Pasche \& Serhan 2004). The major stimulus of CRP synthesis is interleukin 6 (IL6; Goldman \& Liu 1987, Li \& Goldman 1996), which is also responsible for the recruitment of monocytes during chronic inflammation and therefore plays an important role in the transition from acute to chronic inflammation (Gabay 2006). IL1 receptor antagonist (IL1Ra) is secreted by various types of cells including immune cells, epithelial cells, and adipocytes, and is a natural inhibitor of the proinflammatory effect of IL1 $\beta$ (Perrier et al. 2006). Nevertheless, induction of IL1Ra production by other cytokines and acute phase proteins indicates a possible role of this cytokine in chronic inflammation (Arend et al. 1998). Moreover, recent studies implicated IL1Ra in the promotion of type 2 diabetes (Saltevo et al. 2008, Herder et al. 2009), another well-known risk factor for endometrial cancer development (Friberg et al. 2007).

We conducted a case-control study nested within the European Prospective Investigation into Cancer and nutrition (EPIC) to examine the relationships between endometrial cancer risk and blood concentrations of CRP, IL6, and IL1Ra. We also investigated to which extent these inflammatory markers can influence the association of obesity, endogenous sex steroids, and C-peptide (a marker of pancreatic insulin secretion) with endometrial cancer risk. With respect to endometrial cancer, this is the first prospective study of its kind to date, comprising 305 incident cases and 574 matched controls.

\section{Materials and methods}

\section{Study population}

The EPIC cohort is a large, multicenter prospective study, designed to investigate the associations between nutritional, lifestyle, metabolic, and genetic risk factors and cancer incidence. It was initiated in 1992 in ten European countries (Denmark, France, Germany, Greece, Italy, The Netherlands, Norway, Spain, Sweden, and the United Kingdom) and involved about 370000 women and 150000 men. About 246000 women and 140000 men also provided a blood sample. In the present study, Norway was not included because blood samples have been collected only recently, and very few cases of endometrial cancer have been diagnosed after blood donation.
Study population and baseline data collection have been previously described in details (Riboli et al. 2002). In brief, questionnaires included data about dietary, lifestyle and health factors, reproductive history, use of oral contraceptives (OCs) and hormone replacement therapy (HRT), history of any disorders or surgical operations, tobacco smoking and alcohol consumption, occupational history, physical activity, and education level.

\section{Collection and storage of blood samples}

In France, The Netherlands, the United Kingdom, Germany, Spain, Italy, and Greece, blood samples were collected according to a standardized protocol (Riboli et al. 2002). From each subject, about $30 \mathrm{ml}$ of blood was drawn, and serum, plasma, erythrocytes, and buffy coat were aliquoted in plastic straws of $0.5 \mathrm{ml}$ each, which were heat-sealed and stored under liquid nitrogen $\left(-196^{\circ} \mathrm{C}\right)$ in a centralized biobank. In Denmark, blood fractions were aliquoted into $1 \mathrm{ml}$ tubes, and stored in the vapor phase of liquid nitrogen containers $\left(-150^{\circ} \mathrm{C}\right)$. In the Swedish center of Umea, blood samples were divided into ten aliquots of $1.5 \mathrm{ml}$ each (six plasma, two buffy coat, and two erythrocytes), which were rapidly frozen at $-80{ }^{\circ} \mathrm{C}$ in standard freezers.

\section{Determination of menopausal status at blood donation}

Women were considered as premenopausal when they reported menstruating regularly over the past 12 months or, if data were incomplete, when they were $<42$ years of age at recruitment $(99.5 \%$ of EPIC women who had complete data were premenopausal below age 42). Women were considered as postmenopausal when they reported not having had any menses over the past 12 months, or when they reported bilateral ovariectomy. Women who had incomplete data were considered postmenopausal if they were older than 55 . Women who had less than nine menstrual cycles during the year preceding recruitment or women aged between 42 and 55 years and with missing or incomplete questionnaire data or who reported the use of HRT were classified as having peri-menopausal or unknown menopausal status and excluded from the analyses.

\section{Follow-up for cancer incidence and vital status}

Incident cancer cases were identified through several methods, including record linkage with regional cancer registries (Denmark, Sweden, Italy, The Netherlands, Spain, and the United Kingdom), health insurance 
records, cancer and pathology registries, and active follow-up of study subjects (France, Germany, and Greece). Data on vital status were obtained from mortality registries at the regional or national level, in combination with data collected by active follow-up (Greece). For each EPIC center, closure dates of the study period were defined as the latest dates of complete follow-up for both cancer incidence and vital status (dates varied between centers, from June 1999 to December 2003).

\section{Selection of case and control subjects}

Case subjects were selected among women who developed epithelial endometrial cancer after their recruitment into the cohort and before the end of the study period. Cases were coded according to the 10th revision of the International Statistical Classification of Injuries, Disease, and Deaths. Women whose menopausal status was classified as peri-menopausal/ unknown or who used any HRT at the time of blood donation, or any exogenous hormones for contraception or medical purposes, or those with a previous diagnosis of cancer (except non-melanoma skin cancer) were excluded from the study. Women whose endometrial cancers were not primary cancers, or who had a diagnosis of non-epithelial tumors, or who reported hysterectomy were also excluded.

A total of 305 incident cases of epithelial endometrial cancer were identified ( 80 cases among women who were premenopausal at blood donation and 225 cases among women who were postmenopausal at blood donation). The 305 incident cases included 52 cases in Denmark, 49 in Italy, 36 in Spain, 31 in the United Kingdom, 26 in The Netherlands, 8 in Greece, 13 in France, 18 in Germany, and 72 in Sweden.

For each case subject with endometrial cancer, two control subjects were chosen at random among appropriate risk sets consisting of all cohort members alive and free of cancer (except non-melanoma skin cancer) at the time of diagnosis of the index case. An incidence density sampling protocol for control selection was used, such that controls could include subjects who became a case later in time, while each control subject could also be sampled more than once. Matching characteristics for cases and controls included study recruitment center, menopausal status (premenopausal and postmenopausal), age ( \pm 6 months) at enrollment, time of the day of blood collection $( \pm 1 \mathrm{~h})$, fasting status $(<3 \mathrm{~h} ; 3-6 \mathrm{~h}$, and $>6 \mathrm{~h}$ ), and, for premenopausal women, phase of menstrual cycle ('follicular', 'peri-ovulatory', and 'luteal'). As described previously (Kaaks et al. 2005), the menstrual cycle phase was determined using either backward or forward dating, depending on the study center. In centers where both dating methods were used, backward dating was preferred because the length of the luteal phase of the cycle is generally more constant than the length of the follicular phase. With forward dating, the first day of a woman's last period was set to 0 days, and all subsequent days were counted sequentially up to a maximum of 39 days. With backward dating, the first day of a woman's next period after blood donation was set to 28 days, and the date on which a blood sample had been provided was counted backwards from this date to a minimum acceptable value of -11 days. The follicular phase corresponds to days 0 to 11 with forward dating and days -11 to 11 with backward dating; the periovulatory phase corresponds to days 12 to 16 with both backward and forward dating; the luteal phase corresponds to days 17 to 39 with forward dating and days 17 to 28 with backward dating. For the present analysis, 574 controls were selected.

All participants had given their consent for their participation into the EPIC study. The Internal Review Board of IARC and local institutional review boards in participating centers have approved the study.

\section{Laboratory assays}

Serum (or EDTA plasma for Swedish subjects) from cases and matched controls was analyzed within the same analytical batch. CRP and IL6 were measured by using enzyme-linked immunoassays (R\&D Systems Europe, Lille, France) at the International Agency for Research on Cancer (Section of Nutrition and Metabolism), while IL1Ra was measured by beadbased immunoassay (Linco, Millipore, Billerica, MA, USA) at the German Cancer Research Center (Division of Cancer Epidemiology). The different assays were chosen on the basis of a comparative study that was published previously (Dossus et al. 2009b).

For quality control, samples from two sera were analyzed in duplicate within each analytical batch. Mean intra-batch and inter-batch coefficients of variation, calculated on the concentrations from the quality control samples, were 6.8 and $9.3 \%$ for CRP, 6.3 and $8.2 \%$ for IL6, and 15 and $27.7 \%$ for IL1Ra. Samples from cases and controls, which were matched together, were analyzed within the same analytical batch. Twenty-two (2\%) CRP and 428 (50\%) IL1Ra values were below the limit of quantification. These values were set to the limit of quantification $(16 \mathrm{pg} / \mathrm{ml}$ for IL1Ra and $78 \mathrm{ng} / \mathrm{ml}$ for CRP). The remaining missing values (due to problems with analyses or 
limited amount of sample available) represented $<3 \%$ of the samples. Less than $2 \%$ of the samples had values above the highest calibration point for each marker. Owing to these random missing values, CRP was available for 290 complete matched sets of cases and controls (i.e. matched sets with CRP levels available for the case and at least one of the two controls), IL6 for 291, and IL1Ra for 301 complete sets.

Endogenous C-peptide and sex-steroid hormone measurements were performed on a substantial proportion of the study population (225 complete sets) at the International Agency for Research on Cancer, using commercially available immunoassays, and have been described in details elsewhere (Cust et al. 2007, Allen et al. 2008). In brief, serum testosterone, DHEAS, androstenedione, estradiol $\left(\mathrm{E}_{2}\right)$, and estrone $\left(\mathrm{E}_{1}\right)$ were measured by RIAs (Immunotech, Marseille, France and DSL, Webster, TX, USA), while C-peptide and sex hormone-binding globulin (SHBG) were measured by IRMAs (Immunotech and CIS-Bio, Gif-sur-Yvette, France). Serum $E_{2}$ levels were measured only in postmenopausal women because of its large intraindividual variation during the menstrual cycle among premenopausal women.

\section{Statistical analyses}

In all analyses, measurements of inflammatory markers were transformed using the natural logarithm to normalize their distributions.

Pearson's partial correlation coefficients between inflammatory markers and with lifestyle factors were calculated among controls, adjusting for age at blood donation (continuous) and laboratory batch. Univariate associations of baseline characteristics, including BMI (continuous), age at first full-term pregnancy (continuous), number of full-term pregnancies (continuous), age at menarche (continuous), age at menopause (continuous), past OC (yes/no) or HRT use (yes/no), smoking status (never, former, and current smoker), alcohol consumption (continuous), physical activity (inactive, moderately inactive, moderately active, and active), and history of diabetes (yes/no), were evaluated using conditional logistic regression to retain the matching.

Odds ratios (ORs) for endometrial cancer in relation to BMI or circulating inflammatory marker levels were calculated by conditional logistic regression models. IL6 and CRP were categorized into quartiles, and the cut-off points were based on the distributions of the controls. Because about half of the IL1Ra values were below the limit of quantification of the assay, quartiles could not be used for this marker. Therefore, undetectable IL1Ra values were grouped in one category used as the reference category, and values above the limit of quantification were categorized into tertiles (cut-off points based on the distributions of the controls with detectable values). BMI was categorized using the WHO definition of normal weight $\left(<25 \mathrm{~kg} / \mathrm{m}^{2}\right)$, overweight $\left(25-29 \mathrm{~kg} / \mathrm{m}^{2}\right)$, and obese $\left(\geq 30 \mathrm{~kg} / \mathrm{m}^{2}\right)$. Analyses on association between BMI and endometrial cancer risk included only complete matching sets (one case and at least one control) with data on C-peptide, $\mathrm{E}_{1}$, and inflammatory markers (258 cases and 452 controls). Likelihood ratio tests were used to assess linear trends in ORs with assigned quantitative scores 1,2,3, and 4 for the categories. The effects of potential confounders (additionally to the matching criteria, controlled for by design) were examined by including additional terms into the logistic regression models. Potential confounders included BMI, waist circumference, age at menarche, age at menopause (among postmenopausal women only), previous use of OCs, previous use of HRT (among postmenopausal women only), smoking, alcohol consumption, physical activity, and diabetes. Only BMI and waist circumference affected point estimates by more than $10 \%$. Further adjustments for C-peptide, SHBG, testosterone, DHEAS, androstenedione, $\mathrm{E}_{1}$, and postmenopausal $E_{2}$ were also performed.

For analysis of statistical heterogeneity between study countries or between subgroups of menopausal status, age at diagnosis, BMI categories, or lag time between blood donation and diagnosis, ORs were estimated for continuous measurements of inflammatory markers transformed on the $\log _{2}$ scale. In this scale, a unit increase corresponds to a doubling of concentration. Formal tests of heterogeneity between the ORs in different EPIC subgroups were based on $\chi^{2}$-statistics, calculated as the deviations of logistic $\beta$-coefficients observed in each of the subgroups, relative to the overall $\beta$-coefficient.

All statistical tests and corresponding $P$ values were two-sided, and $P$ values $<0.05$ were considered statistically significant. All analyses were performed using the SAS software package (Version 9, SAS Institute, Cary, NC, USA).

\section{Results}

Baseline characteristics of cases and controls are presented in Table 1. On average, women diagnosed with endometrial cancer had a higher BMI than controls $(27.5$ vs $26.0, P<0.0001)$, were more often nulliparous (16.9 vs $7.7 \%, P<0.0001$ ), were older at menopause (50.9 vs 49.8 years, $P=0.002$ ), were less 
Table 1 Baseline characteristics of endometrial cancer cases and matched controls, means (s.D.) or percentages

\begin{tabular}{|c|c|c|c|}
\hline Variable & Cases $(N=305)$ & Controls $(N=574)$ & $P^{a}$ \\
\hline Menopausal status & & & Matched \\
\hline Premenopausal & $26.2 \%$ & $25.1 \%$ & \\
\hline Postmenopausal & $73.8 \%$ & $74.9 \%$ & \\
\hline Age at blood donation (years) & $56.9(7.3)$ & $57.1(7.4)$ & Matched \\
\hline Age at diagnosis (years) & $60.4(7.2)$ & - & - \\
\hline Lag time between blood collection and diagnosis (years) & $3.5(2.6)$ & - & - \\
\hline Body mass index $\left(\mathrm{kg} / \mathrm{m}^{2}\right)$ & $27.5(5.5)$ & $26.0(4.3)$ & $<0.0001$ \\
\hline Obese & $26.2 \%$ & $16.4 \%$ & 0.0002 \\
\hline Waist circumference (cm) & $87.0(13.1)$ & $83.4(11.3)$ & $<0.0001$ \\
\hline Nulliparous & $16.9 \%$ & $7.7 \%$ & $<0.0001$ \\
\hline Number of full-term pregnancies ${ }^{\mathrm{b}}$ & $2.3(1.0)$ & $2.4(1.1)$ & 0.09 \\
\hline Age at first full-term pregnancy (years) ${ }^{b}$ & $24.7(4.2)$ & $24.9(4.3)$ & 0.96 \\
\hline Age at menarche (years) & $13.0(1.5)$ & $13.3(1.6)$ & 0.10 \\
\hline Age at menopause (years) ${ }^{c}$ & $50.9(4.0)$ & $49.8(3.9)$ & 0.002 \\
\hline Previous OC use & $32.8 \%$ & $42.0 \%$ & 0.002 \\
\hline Previous HRT use ${ }^{c}$ & $29.5 \%$ & $16.7 \%$ & 0.0001 \\
\hline Self-reported diabetes & $4.6 \%$ & $3.7 \%$ & 0.50 \\
\hline Physical activity & & & 0.64 \\
\hline Inactive & $26.4 \%$ & $27.1 \%$ & \\
\hline Moderately inactive & $34.6 \%$ & $37.8 \%$ & \\
\hline Moderately active & $23.4 \%$ & $19.5 \%$ & \\
\hline Active & $15.6 \%$ & $15.6 \%$ & \\
\hline Alcohol consumption (g/day) & $7.1(10.6)$ & $7.3(10.3)$ & 0.86 \\
\hline Smoking status & & & 0.18 \\
\hline Never smokers & $66.5 \%$ & $60.7 \%$ & \\
\hline Former smokers & $19.9 \%$ & $22.3 \%$ & \\
\hline Current smokers & $13.5 \%$ & $17.0 \%$ & \\
\hline
\end{tabular}

${ }^{\text {a }} P$ values were calculated by using conditional logistic regression.

${ }^{\mathrm{b}}$ Among parous women only.

${ }^{\mathrm{c} A m o n g}$ postmenopausal women only.

often past OC users (32.8 vs $42.0 \%, P=0.002)$, and more often past HRT users (29.5 vs $16.7 \%, P=0.0001$ ) as reported previously (Dossus et al. 2009a).

Differences in cytokine levels according to baseline characteristics were evaluated among controls after adjusting for age at blood donation, laboratory batch, and BMI. Age at menopause, past HRT and OC use, self-reported diabetes, physical activity, and alcohol consumption were not significantly associated with any of the inflammatory markers (data not shown). However, higher CRP and IL6 mean levels were observed among current smokers (CRP: $1528 \mathrm{ng} / \mathrm{ml}$ and IL6: $1.61 \mathrm{pg} / \mathrm{ml}$ ) compared to past smokers (CRP: $1066 \mathrm{ng} / \mathrm{ml}$ and IL6: $1.38 \mathrm{pg} / \mathrm{ml}$ ) and never smokers (CRP: $1066 \mathrm{ng} / \mathrm{ml}$ and IL6: $1.22 \mathrm{pg} / \mathrm{ml}$ ). IL6 mean levels were also higher in premenopausal women $(1.36 \mathrm{pg} / \mathrm{ml})$ than in postmenopausal women $(1.13 \mathrm{pg} / \mathrm{ml})$. After adjustment for age at blood donation and laboratory batch, there was a relatively strong correlation between IL6 and CRP levels $(r=0.51,95 \%$ confidence interval (CI): $0.44-0.57$; Table 2). Lower degrees of correlation were observed between IL1Ra and IL6 ( $r=0.17$, 95\% CI: 0.09-0.25) or between IL1Ra and CRP $(r=0.23$, 95\% CI: $0.15-0.30)$. Pearson partial correlations with age at blood donation varied between 0.10 for IL1Ra (95\% CI: 0.01-0.18), 0.18 for CRP (95\% CI: 0.10-0.26), and 0.28 for IL6 (95\% CI: $0.20-0.35$ ). All markers were correlated with BMI $(r=0.37,95 \%$ CI: $0.29-0.44$ for CRP; $r=0.32,95 \%$ CI: $0.24-0.39$ for IL6; and $r=0.18,95 \%$ CI: $0.10-0.26$ for IL1Ra) and waist circumference $(r=0.43$, 95\% CI: $0.35-0.50$ for CRP; $r=0.35$, 95\% CI: $0.27-0.43$ for IL6; and $r=0.24,95 \%$ CI: $0.15-0.32$ for IL1Ra). Scatter plots of BMI by inflammatory marker levels for cases and controls are presented in Supplementary Figure 1, see section on supplementary data given at the end of this article. None of the markers correlated with time before centrifugation or storage time of the samples $(r<0.05$, data not shown).

Conditional logistic regression analyses showed an increased risk of endometrial cancer with the three inflammatory markers studied, with an OR of 1.58 (95\% CI: 1.03-2.41) for the highest versus the lowest quartile of CRP, 1.66 (95\% CI: 1.08-2.54) for the highest versus the lowest quartile of IL6, and 1.82 
Table 2 Pearson's partial correlation coefficients (95\% confidence interval $(\mathrm{Cl})$ ) for associations between inflammatory factors, body mass index (BMI), waist circumference, and age at blood donation among controls

\begin{tabular}{lcrr}
\hline & CRP & IL6 & IL1Ra \\
\hline IL6 $^{\mathrm{a}}$ & $0.51(0.44-0.57)$ & & \\
IL1Ra $^{\mathrm{a}}$ & $0.23(0.15-0.30)$ & $0.17(0.09-0.25)$ & $0.32(0.24-0.39)$ \\
BMI $^{\mathrm{a}}$ & $0.37(0.29-0.44)$ & $0.35(0.27-0.43)$ & $0.18(0.10-0.26)$ \\
Waist circumference $^{\mathrm{a}}$ & $0.43(0.35-0.50)$ & $0.28(0.20-0.35)$ & $0.24(0.15-0.32)$ \\
Age at blood donation $^{\mathrm{b}}$ & $0.18(0.10-0.26)$ & $0.10(0.01-0.18)$ \\
\hline
\end{tabular}

${ }^{a}$ Analyses on log-transformed data, adjusted for age at blood donation and laboratory batch.

${ }^{\mathrm{b}}$ Analyses on log-transformed data, adjusted for laboratory batch.

(95\% CI: 1.22-2.73) for the highest versus the lowest category of IL1Ra (Table 3). After adjustment for BMI, these estimates were lower (from $-25 \%$ for IL6 to $-33 \%$ for CRP) and were no longer statistically significant. A similar reduction in risk estimates was also observed after adjustment for waist circumference ( $-19 \%$ for IL1Ra and $-30 \%$ for IL6 and CRP) (data not shown). Further adjustment for C-peptide resulted in an additional risk reduction of 6-12\%, whereas further adjustment for $\mathrm{E}_{1}$ only reduced risk estimate by 0-5\% (Table 4). In postmenopausal women, we observed a slightly stronger effect of the adjustment for $\mathrm{E}_{1}$ on the association between endometrial cancer and CRP (OR in the highest quartile changed from 1.36, $95 \%$ CI: $0.72-2.59$ to $1.21,95 \%$ CI: $0.63-2.34$ ) or IL6 (OR in the highest quartile changed from 1.23, 95\% CI: $0.67-2.25$ to $1.14,95 \%$ CI: $0.62-2.11$ ). Very similar estimates were observed after adjustment for postmenopausal $\mathrm{E}_{2}$ (OR in the highest quartile: 1.23 , 95\% CI: $0.63-2.40$ for CRP and 1.12, 95\%
CI: $0.60-2.10$ for IL6). Adjustments for testosterone, androstenedione, DHEAS, or SHBG did not change risk estimates by more than $5 \%$ (data not shown). Exclusion of subjects with CRP $\geq 10 \mathrm{mg} / \mathrm{l}$ (a level indicative of acute inflammation) did not affect risk estimates (data not shown).

Since half of the subjects had values below the limit of quantification for IL1Ra, we performed sensitivity analyses excluding these subjects. After adjustment for BMI, ORs were 1.23 (95\% CI: 0.61-2.49) for women in the second tertile and 1.92 (95\% CI: 1.02-3.64) for women in the third tertile compared to women in the first tertile of measured IL1Ra $\left(P_{\text {trend }}=0.04\right)$.

No heterogeneity was observed in the associations of CRP and IL1Ra with endometrial cancer risk by country, menopausal status (premenopausal versus postmenopausal), age at diagnosis $(<55$ vs $\geq 55$ years), BMI categories ( $<25,25-29$, and $\geq 30 \mathrm{~kg} / \mathrm{m}^{2}$ ), or lag time between blood donation and diagnosis $(<2$ vs $\geq 2$ years and $<5$ vs $\geq 5$ years) (data not shown).

Table 3 Risk (odds ratio, OR (95\% confidence interval, $\mathrm{Cl})$ ) of endometrial cancer by categories of inflammatory markers

\begin{tabular}{|c|c|c|c|c|c|}
\hline & \multicolumn{4}{|c|}{ Categories } & \multirow[b]{2}{*}{$\boldsymbol{P}_{\text {trend }}{ }^{\mathrm{a}}$} \\
\hline & 1 & 2 & 3 & 4 & \\
\hline \multicolumn{6}{|l|}{ CRP } \\
\hline Quartile cut-offs (ng/ml) ${ }^{\mathrm{b}}$ & $<568$ & $568-1154$ & 1155-2232 & $>2232$ & \\
\hline Cases/controls & $61 / 136$ & $65 / 135$ & $77 / 136$ & $87 / 135$ & \\
\hline Crude (matched) & 1.00 & $1.11(0.73-1.70)$ & $1.34(0.89-2.03)$ & $1.58(1.03-2.41)$ & 0.02 \\
\hline Adjusted for BMI & 1.00 & $0.99(0.65-1.53)$ & $1.14(0.74-1.75)$ & $1.05(0.65-1.69)$ & 0.70 \\
\hline \multicolumn{6}{|l|}{ IL6 } \\
\hline Quartile cut-offs $(\mathrm{pg} / \mathrm{ml})^{\mathrm{b}}$ & $<0.85$ & $0.85-1.21$ & $1.22-1.89$ & $>1.89$ & \\
\hline Cases/controls & $64 / 135$ & $57 / 132$ & $75 / 133$ & $95 / 134$ & \\
\hline Crude (matched) & 1.00 & $0.94(0.61-1.45)$ & $1.27(0.83-1.96)$ & $1.66(1.08-2.54)$ & 0.008 \\
\hline Adjusted for BMI & 1.00 & $0.84(0.54-1.31)$ & $1.05(0.67-1.65)$ & $1.24(0.78-1.98)$ & 0.22 \\
\hline \multicolumn{6}{|l|}{ IL1Ra } \\
\hline Category cut-offs $(\mathrm{pg} / \mathrm{ml})^{\mathrm{b}}$ & $\leq 16.0^{\mathrm{c}}$ & $16.1-59.6$ & $59.7-140.7$ & $>140.7$ & \\
\hline Cases/controls & $142 / 286$ & $33 / 91$ & $51 / 91$ & $75 / 93$ & \\
\hline Crude (matched) & 1.00 & $0.80(0.50-1.27)$ & $1.28(0.83-1.97)$ & $1.82(1.22-2.73)$ & 0.004 \\
\hline Adjusted for BMI & 1.00 & $0.71(0.44-1.14)$ & $1.09(0.70-1.70)$ & $1.44(0.94-2.21)$ & 0.10 \\
\hline
\end{tabular}

${ }^{\text {a }} P$ value for trend with assigned quantitative scores $1,2,3$, and 4 for the categories.

${ }^{b}$ Cut-off points were based on the distribution of controls (of subjects with detectable values).

${ }^{c}$ Values below the limit of quantification. 
Table 4 Risk (odds ratio, OR (95\% confidence interval, CI)) of endometrial cancer by categories of inflammatory markers, in women with C-peptide and estrone measurements

\begin{tabular}{|c|c|c|c|c|c|}
\hline & \multicolumn{4}{|c|}{ Categories } & \multirow[b]{2}{*}{$P_{\text {trend }}$} \\
\hline & 1 & 2 & 3 & 4 & \\
\hline \multicolumn{6}{|l|}{ CRP } \\
\hline Quartile cut-offs (ng/ml) ${ }^{b}$ & $<564$ & $564-1171$ & $1172-2329$ & $>2329$ & \\
\hline Cases/controls & $43 / 99$ & $54 / 97$ & $63 / 99$ & $65 / 98$ & \\
\hline Adjusted for BMI & 1.00 & $1.16(0.71-1.92)$ & $1.25(0.76-2.06)$ & $1.06(0.61-1.85)$ & 0.75 \\
\hline Adjusted for BMI and C-peptide & 1.00 & $1.04(0.62-1.73)$ & $1.07(0.64-1.79)$ & $0.93(0.53-1.64)$ & 0.83 \\
\hline Adjusted for BMI and estrone & 1.00 & $1.17(0.71-1.93)$ & $1.27(0.77-2.10)$ & $1.01(0.58-1.77)$ & 0.86 \\
\hline \multicolumn{6}{|l|}{ IL6 } \\
\hline Quartile cut-offs (pg/ml) ${ }^{b}$ & $<0.85$ & $0.85-1.19$ & $1.20-1.80$ & $>1.80$ & \\
\hline Cases/controls & $46 / 99$ & $45 / 97$ & $60 / 99$ & $74 / 98$ & \\
\hline Adjusted for BMI & 1.00 & $0.91(0.55-1.51)$ & $1.16(0.69-1.97)$ & $1.33(0.78-2.30)$ & 0.20 \\
\hline Adjusted for BMI and C-peptide & 1.00 & $0.87(0.52-1.45)$ & $1.06(0.62-1.82)$ & $1.19(0.68-2.07)$ & 0.40 \\
\hline Adjusted for BMI and estrone & 1.00 & $0.93(0.56-1.56)$ & $1.17(0.69-2.00)$ & $1.30(0.75-2.25)$ & 0.25 \\
\hline \multicolumn{6}{|l|}{ IL1Ra } \\
\hline Category cut-offs (pg/ml) ${ }^{\mathrm{b}}$ & $\leq 16.0^{\mathrm{C}}$ & $16.1-49.5$ & $49.6-120.2$ & $>120.2$ & \\
\hline Cases/controls & $106 / 211$ & $24 / 59$ & $41 / 61$ & $54 / 62$ & \\
\hline Adjusted for BMI & 1.00 & $0.80(0.45-1.43)$ & $1.24(0.74-2.06)$ & $1.56(0.94-2.58)$ & 0.08 \\
\hline Adjusted for BMI and C-peptide & 1.00 & $0.79(0.44-1.40)$ & $1.10(0.65-1.86)$ & $1.47(0.88-2.45)$ & 0.16 \\
\hline Adjusted for BMI and estrone & 1.00 & $0.83(0.47-1.48)$ & $1.24(0.74-2.06)$ & $1.56(0.94-2.60)$ & 0.08 \\
\hline
\end{tabular}

${ }^{\text {a }} P$ value for trend with assigned quantitative scores $1,2,3$, and 4 for the categories.

${ }^{b}$ Cut-off points were based on the distribution of controls (of subjects with detectable values).

${ }^{c}$ Values below the limit of quantification.

For IL6, a stronger risk was observed for a doubling of concentrations among premenopausal women (OR: 1.50, 95\% CI: 1.00-2.24) compared to postmenopausal women (OR: 1.09, 95\% CI: 0.88-1.34; Fig. 1). A similar heterogeneity was observed between cases diagnosed after or before 2 years following blood donation (OR: 1.43, 95\% CI: 1.00-2.04 for cases diagnosed $<2$ years after blood donation and OR: $1.07,95 \%$ CI: $0.86-1.32$ for cases diagnosed more than 2 years after blood donation). However, the heterogeneity tests were not statistically significant $\left(P_{\text {heterogeneity }}=0.16\right.$ and 0.17$)$. Exclusion of cases diagnosed in the first 2 years of follow-up did not modify the heterogeneity observed by menopausal status. Similarly, the heterogeneity observed between early and late diagnosed cases persisted when the analysis was restricted to postmenopausal women.

An OR of 2.02 (95\% CI: $\left.1.26-3.23, P_{\text {trend }}=0.005\right)$ was observed for obese women (BMI $\geq 30 \mathrm{~kg} / \mathrm{m}^{2}$ ) compared to normal weight women (BMI $<25 \mathrm{~kg} / \mathrm{m}^{2}$; Table 5). Adjustments for inflammatory markers resulted in reduction of risk estimates by $11 \%$ for CRP, $15 \%$ for IL6, and $18 \%$ for IL1Ra. After adjustment for C-peptide, obese women had an OR of 1.43 (95\% CI: $0.86-2.40, P_{\text {trend }}=0.21$ ) compared to normal weight women. Adjustment for $\mathrm{E}_{1}$ reduced the OR for obese versus normal weight women to 1.75 (95\% CI: $1.07-2.85, P_{\text {trend }}=0.03$ ). Further adjustment for CRP, IL6, or IL1Ra resulted in a reduction in risk estimates for obese women $(-3$ to $-18 \%)$. In the models adjusted for $\mathrm{C}$-peptide, $\mathrm{E}_{1}$, and inflammatory markers, the ORs for obese versus normal weight women were close to unity (OR: 1.21 , 95\% CI: 0.69-2.14 with further adjustment for CRP; OR: 1.16, 95\% CI: 0.67-2.01 with further adjustment for IL6; OR: $1.10,95 \%$ CI: $0.64-1.90$ with further adjustment for IL1Ra). A similar reduction in the estimates was observed for the risk of endometrial cancer associated with waist circumference after adjustment for inflammatory markers, $\mathrm{E}_{1}$, and $\mathrm{C}$-peptide (data not shown).

\section{Discussion}

In this prospective study, we observed that circulating levels of CRP, IL6, and IL1Ra were significantly associated with endometrial cancer risk, although the association was largely dependent on the levels of adiposity. The association between adiposity measurements and endometrial cancer was also substantially attenuated after adjustment for inflammatory markers, even when the effect of $\mathrm{C}$-peptide or $\mathrm{E}_{1}$ was already taken into account.

One mechanism for the inflammation-mediated association between obesity and endometrial cancer could be the modulation of aromatase activity by cytokines within the adipose tissue (Purohit \& Reed 2002). 


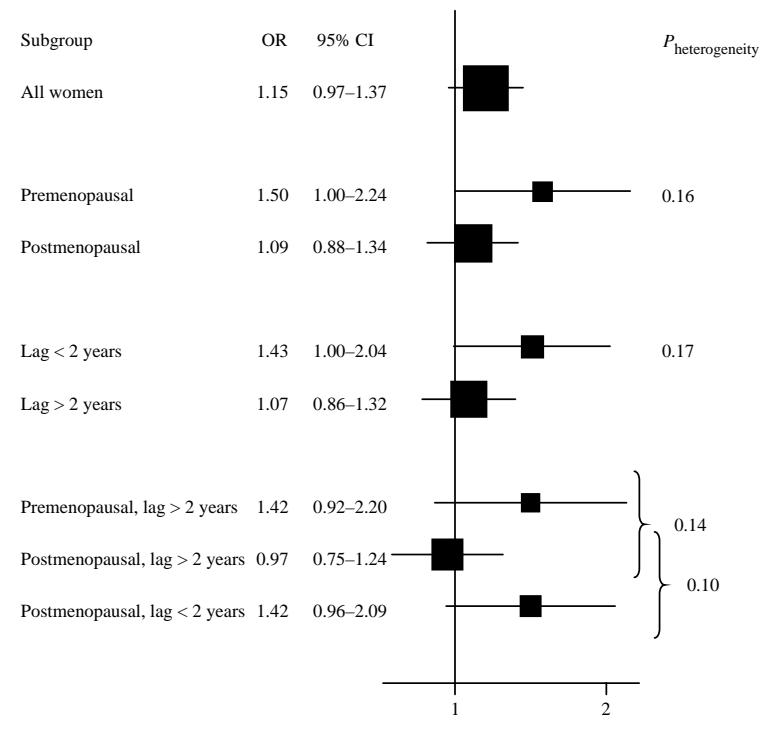

Figure 1 Risk (OR $(95 \% \mathrm{Cl})$ ) of endometrial cancer for IL6 on a continuous $\log _{2}$ scale adjusted for BMI, and stratified by menopausal status and lag time to cancer diagnosis.

After menopause, when the estrogen production from the ovary has ceased, most of the circulating estrogens derive from the peripheral aromatase conversion of androgens in the adipose tissue. It has been shown that IL6 can stimulate aromatase activity in the adipose tissue (Zhao et al. 1995) and therefore enhance the estrogen production and bioavailability. This hypothesis is also supported by the fact that adjustment for inflammatory markers, in addition to $E_{1}$, further attenuated the risk among obese women.

Furthermore, inflammatory markers have also been shown to play an important role in the development of insulin resistance, hyperglycemia, and type 2 diabetes (Greenberg \& McDaniel 2002), which are known risk factors for endometrial cancer (Kaaks et al. 2002). IL6 is one of the major cytokines released by the adipose tissue (Orban et al. 1999, Fain et al. 2004). Its concentration correlates with measures of obesity and insulin resistance (Yudkin et al. 1999, Vozarova et al. 2001), and with the development of type 2 diabetes (Pradhan et al. 2001, Spranger et al. 2003, Hu et al. 2004). Moreover, IL6 enhances the hepatic production of the suppressor of cytokine signaling-3 (a protein that is known to inhibit the insulin signaling pathway) (Lebrun \& Van 2008) and decreases glucose uptake in the adipose tissue (Bastard et al. 2002, Feve \& Bastard 2009). IL6 is also the main regulatory factor of hepatic production of CRP, which also decreases insulin signaling and glucose uptake (Devaraj et al. 2009). IL1Ra is another cytokine that is released in major amounts by the adipose tissue (Juge-Aubry et al. 2003) and is elevated more than sevenfold in obesity (Meier et al. 2002). More recently, this cytokine has also been implicated in the development of type 2 diabetes (Saltevo et al. 2008, Herder et al. 2009). Its role in obesity-related inflammation and in the development of associated diseases is not yet fully understood. However, it has been hypothesized that IL1Ra might have a role in the development of resistance to leptin in obese patients, and this in turn may further sustain obesity (Luheshi et al. 1999, Meier et al. 2002). IL1Ra could also contribute to an increase in adiposity by inhibiting IL1 $\beta$, which inhibits lipogenesis on the one hand, while stimulates lipolysis, glucose transport, and adipocyte maturation in the adipose tissue on the other hand (Juge-Aubry et al. 2003).

It is also possible that cytokines have a direct effect on endometrial carcinogenesis. Within the uterus, IL6 and IL1 are present throughout the menstrual cycle, with a peak of expression during the proliferative phase (Kelly et al. 2001, von Wolff et al. 2002). Cytokines have been shown to enhance the growth and metastasis of various types of tumors (Aggarwal et al. 2006), and alterations in the levels of cytokine production have been reported in several types of cancer, including gynecologic cancers. For example, increased IL6 concentrations have been reported in patients with endometrial carcinoma (Chopra et al. 1997, Punnonen et al. 1998, Bellone et al. 2005, Slater et al. 2006), and laboratory experiments have demonstrated that nuclear factor- $\kappa \mathrm{B}(\mathrm{NF}-\kappa \mathrm{B})$, a cellular transcription factor that activates genes for immune and inflammatory response, is aberrantly expressed in a majority of endometrial cancer tumors (Vaskivuo et al. 2002, Pallares et al. 2004). NF- $\mathrm{BB}$ activation also leads to COX-2 expression, which induces elevated levels of prostaglandin $E_{2}$, a protein that has been shown to promote the transformation of normal endometrium into neoplastic tissue (Jabbour et al. 2006).

The major strengths of our study are the high degree of standardization across countries for blood collection protocols and questionnaire data and the fact that blood samples were collected prospectively prior to endometrial cancer diagnosis. This design reduces the possibility of 'reverse causation bias', which may occur when the presence of a tumor modifies circulating inflammatory marker levels. Indeed, the stronger association observed for IL6 among cases diagnosed in the 2 years following blood collection suggests the presence of a preclinical tumor that can release IL6 (Scambia et al. 1994, Bellone et al. 2005, Slater et al. 2006) and may have influenced circulating levels. However, this was not observed for the other two markers. Another possible explanation for 
Table 5 Risk (odds ratio, OR (95\% confidence interval, $\mathrm{Cl})$ ) of endometrial cancer by categories of body mass index (BMI), with further adjustment for inflammatory markers, C-peptide, and estrone

\begin{tabular}{|c|c|c|c|c|}
\hline \multirow[b]{3}{*}{ Cases/controls } & \multicolumn{3}{|c|}{ BMI WHO categories $\left(\mathrm{kg} / \mathrm{m}^{2}\right)$} & \multirow[b]{2}{*}{$P_{\text {trend }}{ }^{\mathrm{a}}$} \\
\hline & $<25$ & 25-29 & $30+$ & \\
\hline & $81 / 174$ & $82 / 151$ & $61 / 68$ & \\
\hline Crude (matched) & 1.00 & $1.23(0.82-1.84)$ & $2.02(1.26-3.23)$ & 0.005 \\
\hline Adjusted for estrone & 1.00 & $1.14(0.75-1.72)$ & $1.75(1.07-2.85)$ & 0.03 \\
\hline Adjusted for C-peptide & 1.00 & $1.02(0.67-1.56)$ & $1.43(0.86-2.40)$ & 0.21 \\
\hline Adjusted for C-peptide and estrone & 1.00 & $0.94(0.61-1.46)$ & $1.24(0.73-2.12)$ & 0.49 \\
\hline Adjusted for CRP & 1.00 & $1.15(0.76-1.76)$ & $1.79(1.06-3.02)$ & 0.04 \\
\hline Adjusted for estrone and CRP & 1.00 & $1.08(0.70-1.66)$ & $1.57(0.92-2.69)$ & 0.12 \\
\hline Adjusted for C-peptide and CRP & 1.00 & $1.00(0.65-1.55)$ & $1.39(0.80-2.41)$ & 0.29 \\
\hline Adjusted for C-peptide, estrone, and CRP & 1.00 & $0.93(0.60-1.46)$ & $1.21(0.69-2.14)$ & 0.57 \\
\hline Adjusted for IL6 & 1.00 & $1.14(0.76-1.73)$ & $1.72(1.04-2.85)$ & 0.04 \\
\hline Adjusted for estrone and IL6 & 1.00 & $1.06(0.70-1.62)$ & $1.51(0.90-2.53)$ & 0.15 \\
\hline Adjusted for C-peptide and IL6 & 1.00 & $0.99(0.64-1.52)$ & $1.33(0.78-2.27)$ & 0.35 \\
\hline Adjusted for C-peptide, estrone, and IL6 & 1.00 & $0.92(0.59-1.43)$ & $1.16(0.67-2.01)$ & 0.68 \\
\hline Adjusted for IL1Ra & 1.00 & $1.15(0.76-1.73)$ & $1.65(1.01-2.71)$ & 0.05 \\
\hline Adjusted for estrone and IL1Ra & 1.00 & $1.07(0.70-1.62)$ & $1.45(0.87-2.41)$ & 0.18 \\
\hline Adjusted for C-peptide and IL1Ra & 1.00 & $0.99(0.64-1.51)$ & $1.26(0.74-2.14)$ & 0.44 \\
\hline Adjusted for C-peptide, estrone, and IL1Ra & 1.00 & $0.91(0.59-1.42)$ & $1.10(0.64-1.90)$ & 0.80 \\
\hline
\end{tabular}

${ }^{\text {a }} P$ value for trend with assigned quantitative scores 1,2 , and 3 for the categories.

the stronger association with IL6 in the first years of follow-up could be that IL6 levels at recruitment may not be predictive of inflammatory status after several years of follow-up during which major determinants of IL6 levels such as smoking status, physical activity, or medical treatments might have changed.

One limitation of our study is that only one cytokine measurement, at a single point in time, was available to reflect long-term exposure to chronic inflammation. This could result in random misclassification, in view of intra-individual variations over time, and therefore may have led to underestimation of risk estimates. However, a recent study measuring cytokine levels in repeated blood samples taken over a 2-year period showed moderate to good reliability with correlations in the range of $0.57-0.92$ for the inflammatory markers included in our study (Gu et al. 2009). The latter results suggest that, while random misclassification certainly occurs, its attenuating effects on risk estimates are moderate. Another limitation is the fact that about half of the values for IL1Ra were below the limit of quantification of the assay. Results for this marker should therefore be considered with caution until reproduced in other studies.

In conclusion, elevated prediagnostic levels of the inflammatory markers CRP, IL6, and IL1Ra were associated with an increased risk of endometrial cancer. Our results provide some degree of support to the hypothesis that chronic inflammation, through its association with insulin resistance and estrogen production, but also independently of these two pathways, might mediate the obesity-related increase in risk of endometrial cancer, although after adjustment for BMI the associations were weaker and no longer statistically significant. To our knowledge, this is the first study of its kind, and further prospective studies are needed to confirm these observations. Further experimental studies should also investigate whether inflammation has a direct and independent effect on endometrial carcinogenesis.

\section{Supplementary data}

This is linked to the online version of the paper at http://dx. doi.org/10.1677/ERC-10-0053.

\section{Declaration of interest}

The authors declare that there is no conflict of interest that could be perceived as prejudicing the impartiality of the research reported.

\section{Funding}

This work was supported by the Word Cancer Research Fund (grant number 2007/13). The EPIC study was funded by 'Europe Against Cancer' Programme of the European Commission (SANCO); Ligue contre le Cancer (France); Société 3M (France); Mutuelle Générale de l'Education Nationale; Institut National de la Santé et de la Recherche 
Médicale (INSERM); German Cancer Aid; German Cancer Research Centre; German Federal Ministry of Education and Research; Danish Cancer Society; ISCIII (RETICC DR06/0020) of the Spanish Ministry of Health and the participating regional governments and institutions of Spain; Cancer Research UK; Medical Research Council, UK; Hellenic Ministry of Health; Stavros Niarchos Foundation; Hellenic Health Foundation; Italian Association for Research on Cancer; Italian National Research Council; Dutch Ministry of Public Health, Welfare, and Sports; Dutch Ministry of Health; Dutch Prevention Funds; LK Research Funds; Dutch ZON (Zorg Onderzoek Nederland); World Cancer Research Fund (WCRF); Swedish Cancer Society; Swedish Scientific Council; Regional Government of Skane, Sweden; Norwegian Cancer Society.

\section{Acknowledgements}

We thank Miss Britta Lederer, Miss Sigrid Henke, and Mr Thomas Cler for their meticulous work in performing the immunoassays and Mr David Achaintre for the supervision of the laboratory work. We also thank the technical support of the IARC Biological Resource Center for sample retrieval and processing.

\section{References}

Aggarwal BB, Shishodia S, Sandur SK, Pandey MK \& Sethi G 2006 Inflammation and cancer: how hot is the link? Biochemical Pharmacology 72 1605-1621. (doi:10.1016/j.bcp.2006.06.029)

Allen NE, Key TJ, Dossus L, Rinaldi S, Cust A, Lukanova A, Peeters PH, Onland-Moret NC, Lahmann PH, Berrino F et al. 2008 Endogenous sex hormones and endometrial cancer risk in women in the European Prospective Investigation into Cancer and Nutrition (EPIC). Endocrine-Related Cancer 15 485-497. (doi:10.1677/ ERC-07-0064)

Arend WP, Malyak M, Guthridge CJ \& Gabay C 1998 Interleukin-1 receptor antagonist: role in biology. Annual Review of Immunology 16 27-55. (doi:10.1146/annurev. immunol.16.1.27)

Bastard JP, Maachi M, Van Nhieu JT, Jardel C, Bruckert E, Grimaldi A, Robert JJ, Capeau J \& Hainque B 2002 Adipose tissue IL- 6 content correlates with resistance to insulin activation of glucose uptake both in vivo and in vitro. Journal of Clinical Endocrinology and Metabolism 87 2084-2089. (doi:10.1210/jc.87.5.2084)

Bellone S, Watts K, Cane' S, Palmieri M, Cannon MJ, Burnett A, Roman JJ, Pecorelli S \& Santin AD 2005 High serum levels of interleukin-6 in endometrial carcinoma are associated with uterine serous papillary histology, a highly aggressive and chemotherapy-resistant variant of endometrial cancer. Gynecologic Oncology 98 92-98. (doi:10.1016/j.ygyno.2005.03.016)
Calle EE \& Kaaks R 2004 Overweight, obesity and cancer: epidemiological evidence and proposed mechanisms.

Nature Reviews. Cancer 4 579-591. (doi:10.1038/nrc1408)

Chopra V, Dinh TV \& Hannigan EV 1997 Serum levels of interleukins, growth factors and angiogenin in patients with endometrial cancer. Journal of Cancer Research and Clinical Oncology 123 167-172. (doi:10.1007/ s004320050045)

Coussens LM \& Werb Z 2002 Inflammation and cancer. Nature 420 860-867. (doi:10.1038/nature01322)

Cust AE, Allen NE, Rinaldi S, Dossus L, Friedenreich C, Olsen A, Tjonneland A, Overvad K, Clavel-Chapelon F, Boutron-Ruault MC et al. 2007 Serum levels of C-peptide, IGFBP-1 and IGFBP-2 and endometrial cancer risk; results from the European prospective investigation into cancer and nutrition. International Journal of Cancer 120 2656-2664. (doi:10.1002/ijc.22578)

Das UN 2001 Is obesity an inflammatory condition? Nutrition 17 953-966. (doi:10.1016/S08999007(01)00672-4)

Devaraj S, Singh U \& Jialal I 2009 Human C-reactive protein and the metabolic syndrome. Current Opinion in Lipidology 20 182-189. (doi:10.1097/MOL. 0b013e32832ac03e)

Dossus L, Allen N, Kaaks R, Bakken K, Lund E, Tjonneland A, Olsen A, Overvad K, Clavel-Chapelon F, Fournier A et al. 2009a Reproductive risk factors and endometrial cancer: the European prospective investigation into cancer and nutrition. International Journal of Cancer 127 442-451. (doi:10.1002/ijc.25050)

Dossus L, Becker S, Achaintre D, Kaaks R \& Rinaldi S $2009 \mathrm{~b}$ Validity of multiplex-based assays for cytokine measurements in serum and plasma from "non-diseased" subjects: comparison with ELISA. Journal of Immunological Methods 350 125-132. (doi:10.1016/ j.jim.2009.09.001)

Ehrmann DA, Barnes RB \& Rosenfield RL 1995 Polycystic ovary syndrome as a form of functional ovarian hyperandrogenism due to dysregulation of androgen secretion. Endocrine Reviews 16 322-353. (doi:10.1210/edrv-16-3-322)

Fain JN, Madan AK, Hiler ML, Cheema P \& Bahouth SW 2004 Comparison of the release of adipokines by adipose tissue, adipose tissue matrix, and adipocytes from visceral and subcutaneous abdominal adipose tissues of obese humans. Endocrinology 145 2273-2282. (doi:10.1210/en. 2003-1336)

Feve B \& Bastard JP 2009 The role of interleukins in insulin resistance and type 2 diabetes mellitus. Nature Reviews. Endocrinology 5 305-311. (doi:10.1038/nrendo.2009.62)

Franks S 1995 Polycystic ovary syndrome. New England Journal of Medicine 333 853-861. (doi:10.1056/ NEJM199509283331307)

Friberg E, Orsini N, Mantzoros CS \& Wolk A 2007 Diabetes mellitus and risk of endometrial cancer: a meta-analysis. Diabetologia 50 1365-1374. (doi:10.1007/s00125-0070681-5) 
Gabay C 2006 Interleukin-6 and chronic inflammation. Arthritis Research \& Therapy 8 (Supplement 2) S3. (doi:10.1186/ar1917)

Goldman ND \& Liu TY 1987 Biosynthesis of human C-reactive protein in cultured hepatoma cells is induced by a monocyte factor(s) other than interleukin-1. Journal of Biological Chemistry 262 2363-2368.

Greenberg AS \& McDaniel ML 2002 Identifying the links between obesity, insulin resistance and beta-cell function: potential role of adipocyte-derived cytokines in the pathogenesis of type 2 diabetes. European Journal of Clinical Investigation 32 (Supplement 3) 24-34. (doi:10.1046/j.1365-2362.32.s3.4.x)

Gu Y, Zeleniuch-Jacquotte A, Linkov F, Koenig KL, Liu M, Velikokhatnaya L, Shore RE, Marrangoni A, Toniolo P, Lokshin AE et al. 2009 Reproducibility of serum cytokines and growth factors. Cytokine 45 44-49. (doi:10. 1016/j.cyto.2008.10.014)

Herder C, Brunner EJ, Rathmann W, Strassburger K, Tabak AG, Schloot NC \& Witte DR 2009 Elevated levels of the anti-inflammatory interleukin-1 receptor antagonist precede the onset of type 2 diabetes: the Whitehall II study. Diabetes Care 32 421-423. (doi:10.2337/dc08-1161)

Hu FB, Meigs JB, Li TY, Rifai N \& Manson JE 2004 Inflammatory markers and risk of developing type 2 diabetes in women. Diabetes 53 693-700. (doi:10.2337/ diabetes.53.3.693)

International Agency for Research on Cancer (WHO) 2002 IARC Handbooks of Cancer Prevention. Weight Control and Physical Activity. Lyon: IARC Press.

Jabbour HN, Sales KJ, Smith OP, Battersby S \& Boddy SC 2006 Prostaglandin receptors are mediators of vascular function in endometrial pathologies. Molecular and Cellular Endocrinology 252 191-200. (doi:10.1016/j. mce.2006.03.025)

Juge-Aubry CE, Somm E, Giusti V, Pernin A, Chicheportiche R, Verdumo C, Rohner-Jeanrenaud F, Burger D, Dayer JM \& Meier CA 2003 Adipose tissue is a major source of interleukin-1 receptor antagonist: upregulation in obesity and inflammation. Diabetes 52 1104-1110. (doi:10.2337/diabetes.52.5.1104)

Kaaks R, Lukanova A \& Kurzer MS 2002 Obesity, endogenous hormones, and endometrial cancer risk: a synthetic review. Cancer Epidemiology, Biomarkers \& Prevention 11 1531-1543.

Kaaks R, Berrino F, Key T, Rinaldi S, Dossus L, Biessy C, Secreto G, Amiano P, Bingham S, Boeing H et al. 2005 Serum sex steroids in premenopausal women and breast cancer risk within the European Prospective Investigation into Cancer and Nutrition (EPIC). Journal of the National Cancer Institute 97 755-765. (doi:10.1093/jnci/dji132)

Kelly RW, King AE \& Critchley HO 2001 Cytokine control in human endometrium. Reproduction 121 3-19. (doi:10. 1530/rep.0.1210003)

Key TJ \& Pike MC 1988 The dose-effect relationship between 'unopposed' oestrogens and endometrial mitotic rate: its central role in explaining and predicting endometrial cancer risk. British Journal of Cancer 57 205-212. (doi:10.1038/bjc.1988.44)

Lebrun P \& Van OE 2008 SOCS proteins causing trouble in insulin action. Acta Physiologica 192 29-36. (doi:10. 1111/j.1748-1716.2007.01782.x)

Li SP \& Goldman ND 1996 Regulation of human C-reactive protein gene expression by two synergistic IL-6 responsive elements. Biochemistry 35 9060-9068. (doi:10.1021/ bi953033d)

Luheshi GN, Gardner JD, Rushforth DA, Loudon AS \& Rothwell NJ 1999 Leptin actions on food intake and body temperature are mediated by IL-1. PNAS 96 7047-7052. (doi:10.1073/pnas.96.12.7047)

Meier CA, Bobbioni E, Gabay C, Assimacopoulos-Jeannet F, Golay A \& Dayer JM 2002 IL-1 receptor antagonist serum levels are increased in human obesity: a possible link to the resistance to leptin? Journal of Clinical Endocrinology and Metabolism 87 1184-1188. (doi:10.1210/jc.87.3.1184)

Modugno F, Ness RB, Chen C \& Weiss NS 2005 Inflammation and endometrial cancer: a hypothesis. Cancer Epidemiology, Biomarkers \& Prevention 14 2840-2847. (doi:10.1158/1055-9965.EPI-05-0493)

Mohamed-Ali V, Goodrick S, Rawesh A, Katz DR, Miles JM, Yudkin JS, Klein S \& Coppack SW 1997 Subcutaneous adipose tissue releases interleukin-6, but not tumor necrosis factor-alpha, in vivo. Journal of Clinical Endocrinology and Metabolism 82 4196-4200. (doi:10.1210/jc.82.12.4196)

Orban Z, Remaley AT, Sampson M, Trajanoski Z \& Chrousos GP 1999 The differential effect of food intake and beta-adrenergic stimulation on adipose-derived hormones and cytokines in man. Journal of Clinical Endocrinology and Metabolism 84 2126-2133. (doi:10.1210/jc.84.6.2126)

Pallares J, Martinez-Guitarte JL, Dolcet X, Llobet D, Rue M, Palacios J, Prat J \& Matias-Guiu X 2004 Abnormalities in the NF-kappaB family and related proteins in endometrial carcinoma. Journal of Pathology 204 569-577. (doi:10. 1002/path.1666)

Pasche B \& Serhan CN 2004 Is C-reactive protein an inflammation opsonin that signals colon cancer risk? Journal of the American Medical Association 291 623-624. (doi:10.1001/jama.291.5.623)

Perrier S, Darakhshan F \& Hajduch E 2006 IL-1 receptor antagonist in metabolic diseases: Dr Jekyll or Mr Hyde? FEBS Letters 580 6289-6294. (doi:10.1016/j.febslet. 2006.10.061)

Pradhan AD, Manson JE, Rifai N, Buring JE \& Ridker PM 2001 C-reactive protein, interleukin 6, and risk of developing type 2 diabetes mellitus. Journal of the American Medical Association 286 327-334. (doi:10.1001/jama.286.3.327)

Punnonen R, Teisala K, Kuoppala T, Bennett B \& Punnonen J 1998 Cytokine production profiles in the peritoneal fluids of patients with malignant or benign 
gynecologic tumors. Cancer 83 788-796. (doi:10.1002/ (SICI)1097-0142(19980815)83:4<788::AID-

CNCR24>3.0.CO;2-N)

Purohit A \& Reed MJ 2002 Regulation of estrogen synthesis in postmenopausal women. Steroids $\mathbf{6 7}$ 979-983. (doi:10.1016/S0039-128X(02)00046-6)

Reeves GK, Pirie K, Beral V, Green J, Spencer E \& Bull D 2007 Cancer incidence and mortality in relation to body mass index in the Million Women Study: cohort study. BMJ 335 1134. (doi:10.1136/bmj.39367.495995.AE)

Riboli E, Hunt KJ, Slimani N, Ferrari P, Norat T, Fahey M, Charrondiere UR, Hemon B, Casagrande C, Vignat J et al. 2002 European Prospective Investigation into Cancer and Nutrition (EPIC): study populations and data collection. Public Health Nutrition 5 1113-1124. (doi:10.1079/ PHN2002394)

Salamonsen LA 2003 Tissue injury and repair in the female human reproductive tract. Reproduction 125 301-311. (doi:10.1530/rep.0.1250301)

Saltevo J, Laakso M, Jokelainen J, Keinanen-Kiukaanniemi S, Kumpusalo E \& Vanhala M 2008 Levels of adiponectin, C-reactive protein and interleukin-1 receptor antagonist are associated with insulin sensitivity: a population-based study. Diabetes/Metabolism Research and Reviews $\mathbf{2 4}$ 378-383. (doi:10.1002/dmrr.831)

Scambia G, Testa U, Panici PB, Martucci R, Foti E, Petrini M, Amoroso M, Masciullo V, Peschle C \& Mancuso S 1994 Interleukin-6 serum levels in patients with gynecological tumors. International Journal of Cancer 57 318-323. (doi:10.1002/ijc.2910570305)

Slater M, Cooper M \& Murphy CR 2006 Human growth hormone and interleukin- 6 are upregulated in endometriosis and endometrioid adenocarcinoma. Acta Histochemica 108 13-18. (doi:10.1016/j.acthis. 2006.01.004)

Spranger J, Kroke A, Mohlig M, Hoffmann K, Bergmann MM, Ristow M, Boeing H \& Pfeiffer AF 2003 Inflammatory cytokines and the risk to develop type 2 diabetes: results of the prospective population-based European Prospective Investigation into Cancer and Nutrition (EPIC)-Potsdam Study. Diabetes 52 812-817. (doi:10.2337/diabetes.52.3.812)

Vaskivuo TE, Stenback F \& Tapanainen JS 2002 Apoptosis and apoptosis-related factors Bcl-2, Bax, tumor necrosis factor-alpha, and NF-kappaB in human endometrial hyperplasia and carcinoma. Cancer 95 1463-1471. (doi:10.1002/cncr.10876)

Visser M, Bouter LM, McQuillan GM, Wener MH \& Harris TB 1999 Elevated C-reactive protein levels in overweight and obese adults. Journal of the American Medical Association 282 2131-2135. (doi:10.1001/jama.282.22.2131)

Vozarova B, Weyer C, Hanson K, Tataranni PA, Bogardus C \& Pratley RE 2001 Circulating interleukin-6 in relation to adiposity, insulin action, and insulin secretion. Obesity Research 9 414-417. (doi:10.1038/oby.2001.54)

von Wolff M, Stieger S, Lumpp K, Bucking J, Strowitzki T \& Thaler CJ 2002 Endometrial interleukin-6 in vitro is not regulated directly by female steroid hormones, but by pro-inflammatory cytokines and hypoxia. Molecular Human Reproduction 8 1096-1102. (doi:10.1093/molehr/ 8.12.1096)

World Cancer Research Fund/American Institute for Cancer Research 2007 Food, Nutrition, Physical Activity, and the Prevention of Cancer: a Global Perspective. Washington DC: AICR.

Yudkin JS, Stehouwer CD, Emeis JJ \& Coppack SW 1999 C-reactive protein in healthy subjects: associations with obesity, insulin resistance, and endothelial dysfunction: a potential role for cytokines originating from adipose tissue? Arteriosclerosis, Thrombosis, and Vascular Biology 19 972-978.

Zhao Y, Nichols JE, Bulun SE, Mendelson CR \& Simpson ER 1995 Aromatase P450 gene expression in human adipose tissue. Role of a Jak/STAT pathway in regulation of the adipose-specific promoter. Journal of Biological Chemistry 270 16449-16457. (doi:10.1074/jbc.270.27.16449) 